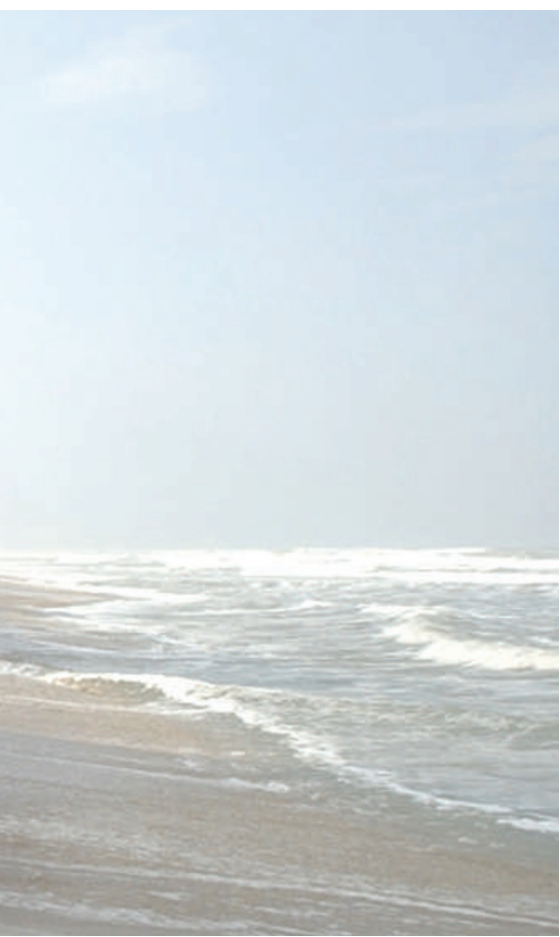

homeowners in California because of the growing risk of wildfires there.

With decades of experience in risk assessment, insurance companies and the consulting firms that they use remain best-placed to evaluate a place's environmental hazards, says Celine Herweijer, principal climate scientist for Risk Management Solutions in London. She adds that although the idea of giving the public risk information is a good one, the information Climate Appraisal is currently selling will be of limited use to the homeowner.

In the case of floods, for example, homeowners would benefit most from estimates of the probability of a flood of a certain depth, Herweijer says, rather than from a national map of flood events, or a list of past floods in their area.

By linking climate change to someone's home address, Climate Appraisal is entering new and fairly treacherous waters. All in all, however, Herweijer and other climate scientists say the idea of making local risk information more widely available is valuable. In attempting to work out what to present and how to present it, most agree that the venture should be admired for its ambition. "You have to applaud it for what it is trying to do," says Schmidt.

Lucy Odling-Smee

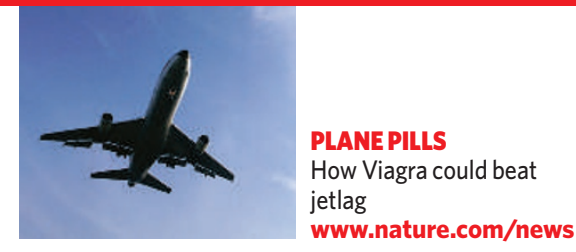

\title{
Help flies in for human genome
}

Worms and flies are to be enlisted by researchers attempting to make sense of the instructions embedded in the human genome.

Since 2003, geneticists in the United States have been engaged in the pilot phase of a project called ENCODE - the Encyclopedia of DNA elements - which aims to catalogue all the functional parts of the human genome at a cost of around US $\$ 20$ million a year.

Now, a four-year, $\$ 57$ million project called modENCODE will add roundworms (Caenorhabditis elegans) and fruitflies (Drosophila melanogaster) to the mix. By analysing these trusty model organisms, scientists hope to make better sense of the human-genome data gleaned from ENCODE.

Grants from modENCODE will support scientists who are cataloguing the major types of functional genetic elements. These include RNAs that cut genetic transcripts into different genes and fine-tune gene expression, modifications to bundles of protein and DNA called chromatin, and DNA sequences that control how and when genes are transcribed.

Researchers say that the

Analysing the fruitfly could help researchers uncover the secrets of the human genome.

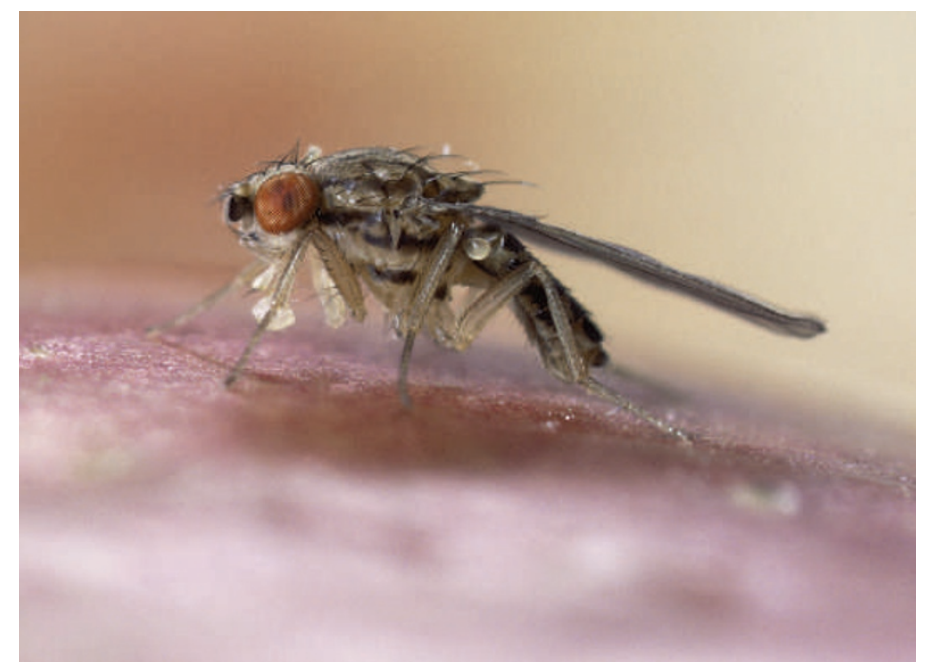

project, which was announced by the US National Human Genome Research Institute (NHGRI) on 14 May, is needed because ENCODE has shown that the human genome is even more complex than they originally thought (see Nature 441, 398-401; 2006).

ENCODE's pilot phase genome and has generated a slew of techniques for analysing a portfolio of technologies that can be applied with high specificity and sensitivity to decode the parts list of the human genome at a rather precise level," says Francis Collins, the NHGRI's director.

So far, the project has revealed that genes are regulated by complicated networks that span huge portions of the genome; that RNA plays a previously unappreciated part in determining how proteins are made from genes; and that scientists don't fully understand some of the regulatory motifs that they have been studying for years.

Scientists hope that modENCODE will enable them to work out these processes by looking at the model covered just $1 \%$ of the human the genome. "We now have organisms, which have much smaller genomes than humans and are easier to manipulate in the lab. The roundworm has about 100 million base pairs in its genome and the fruitfly 180 million, compared with 3 billion base pairs found in the human genome.

"These are hard issues, and without the experimental feedback you can get from worms and flies, it's hard to see when we're really going to touch ground," says Robert Waterston of the University of Washington in Seattle and recipient of a \$5.4-million modENCODE grant to study C. elegans.

But at the end of modENCODE's first four years, will scientists be able to pat themselves on the back and move on to something else? It's unlikely, they say, given that human genetics seems to evolve into a more complicated subject with every new foray into the genome.

"We should get a lot closer," says Lincoln Stein of Cold Spring Harbor Laboratory in New York, and the head of a new centre to coordinate the modENCODE data. "If we can't nail it down, we'll at least have a lot of it thumbtacked to the wall." Erika Check 